\title{
Current status of the laser guide star upgrade at Subaru Telescope
}

Etsuko Mieda, Yoko Tanaka, Matthew Wung, Yosuke Minowa, Christophe Clergeon, et al.

Etsuko Mieda, Yoko Tanaka, Matthew Wung, Yosuke Minowa, Christophe Clergeon, Yoshito Ono, Takashi Hattori, Yutaka Hayano, Masayuki Akiyama, Francois Rigaut, Celine d'Orgeville, "Current status of the laser guide star upgrade at Subaru Telescope," Proc. SPIE 10703, Adaptive Optics Systems VI, 107033P (12 July 2018); doi: 10.1117/12.2314093

Event: SPIE Astronomical Telescopes + Instrumentation, 2018, Austin, Texas, United States 


\title{
Current status of the Laser Guide Star Upgrade at Subaru Telescope
}

\author{
Etsuko Mieda ${ }^{\mathrm{a}}$, Yoko Tanaka ${ }^{\mathrm{a}}$, Matthew Wunga ${ }^{\mathrm{a}}$, Yosuke Minowa ${ }^{\mathrm{a}}$, Christophe Clergeon ${ }^{\mathrm{a}}$, \\ Yoshito Ono $^{\mathrm{a}}$, Takashi Hattori ${ }^{\mathrm{a}}$, Yutaka Hayano ${ }^{\mathrm{b}}$, Masayuki Akiyama ${ }^{\mathrm{c}}$, Francois Rigaut ${ }^{\mathrm{d}}$, and \\ Celine d'Orgeville ${ }^{\mathrm{d}}$

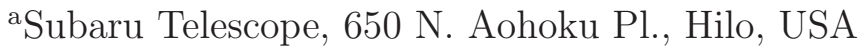 \\ ${ }^{b}$ National Astronomical Observatory of Japan, 2-21-1 Osawa, Mitaka, Japan

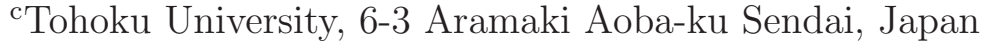 \\ dAustralian National University, Canberra ACT 0200, Australia
}

\begin{abstract}
We report the current status of the laser guide star upgrade at Subaru Telescope with a new, more powerful TOPTICA/MPBC laser. While we recycle many of our existing components, such as laser launch telescope, we need to design a new mirror-based laser relay system to replace the current fiber-based relay to accommodate the high power beam. The laser unit has been delivered to Subaru office in March 2018 and installed in a testing lab in June 2018. We describe the preliminary design and its requirements and report future plans. This upgrade will not only improve our current adaptive optics system but also be the first step toward the future laser tomography and ground layer adaptive optics system at Subaru Telescope.
\end{abstract}

Keywords: Adaptive Optics, Laser Guide Star

\section{INTRODUCTION}

Subaru Telescope is a $8.2 \mathrm{~m}$ telescope located on Mauna Kea in Hawaii. It has been one of the leading facilities for optical to infrared astronomy for almost 20 years. With the help of an adaptive optics (AO) system, we utilize the benefit of a large aperture and study a wide range of topics, from high redshift galaxies to exoplanets around nearby stars (e.g., Ref. 1, 2).

Our AO system, called AO188, ${ }^{3}$ is installed on the Nasmyth Infrared platform (NsIR) and consists of a high order 188 element curvature wavefront sensor (CWFS), a low order $2 \times 2$ Shack-Hartmann (SH) WFS (both WFSs at $1000 \mathrm{~Hz}$ ), and a bimorph deformable mirror (DM). Both high and low order WFSs use avalanche photodiodes (APDs) as detectors. Incoming ligh is split into $0.45 \leq \lambda \leq 0.9 \mu \mathrm{m}$ and $0.9 \leq \lambda \leq 5.3 \mu \mathrm{m}$ to be used for wavefront sensing and science, respectively. AO188 supports natural guide star (NGS) and laser guide star (LGS) modes. Our AO system is currently under a major upgrade process (Ref. 4,5), and a laser upgrade is one of the initial steps toward the ultimate AO facility at Subaru (see $\S 3$ for future plans).

Our current LGS is a sodium $\left(\mathrm{Na}_{2}, \lambda=589.159 \mathrm{~nm}\right.$ ) laser created by the sum-frequency generation technique, where two $(\lambda=1064 \mathrm{~nm}$ and $\lambda=1319 \mathrm{~nm})$ mode-locked Nd:YAG lasers are combined in a nonlinear optical crystal. ${ }^{6}$ The laser unit is placed in a clean, temperature controlled room on NsIR, and the beam is transferred to a laser launch telescope (LLT) behind the secondary mirror by a single mode fiber relay system. LGS mode observation has been open for science use since 2011 and used for more than 300 nights. Please see Ref. 7 and reference therein for technical details and operation performance of our current laser system.

Initially the laser produced $5.4 \mathrm{~W}$ on average $(6.8 \mathrm{~W}$ maximum) output power in the laser room, or $2.4 \mathrm{~W}$ on-sky (11.7 mag in R) after going through the relay fibers and optics; however, as time goes by, the laser power is getting unstable and slowly becomes lower (Figure 1). This deterioration is mainly caused by the loss of the relay fiber transmittance.

Send correspondence to Etsuko Mieda: mieda@naoj.org 
To replace the current laser with more powerful and easy operation laser, Subaru decided to upgrade the laser facility with a TOPTICA/MPBC laser, following the huge success with their new generation of lasers at Very Large Telescope (VLT) and Keck Telescope. ${ }^{8}$ TOPTICA/MPBC laser uses narrow-band Raman Fiber Amplifier (EFRA) technology to generate 20 to $25 \mathrm{~W}$ continuous wave at $589 \mathrm{~nm}$ (Ref. 9). The laser unit is composed of two parts: electronics cabinet (EC) and laser head (LH), shown in Figure 2. Unlike the previous generation of lasers, including Subaru's, both parts do not need to securely sit in a clean room and can sustain under a varying gravity. At Subaru, the EC will sit on NsIR, and the LH will be installed on the front side of the telescope center section. This flexible installation capability reduces the required space and let us improve other instrument environment on NsIR.

While we have already received more powerful laser and plan to recycle many existing components, we cannot simply replace our current laser unit with this new TOPTICA/MPBC laser unit because of its high power. We have to redesign a new mirror-based relay system to transfer the beam to the LLT. In this proceeding, we split the new design into four sections and discuss requirements of each section in $\S 2$. In $\S 3$, we summarize our current status and report the future schedule and plans.

\section{NEW DESIGN OVERVIEW AND REQUIREMENTS}

A new Subaru LGS facility configuration consists of four sections: 1) TOPTICA/MPBC EC and its heat exchange unit (HEU) on NsIR, 2) TOPTICA/MPBC LH and an optical bench on a side of the telescope center section,
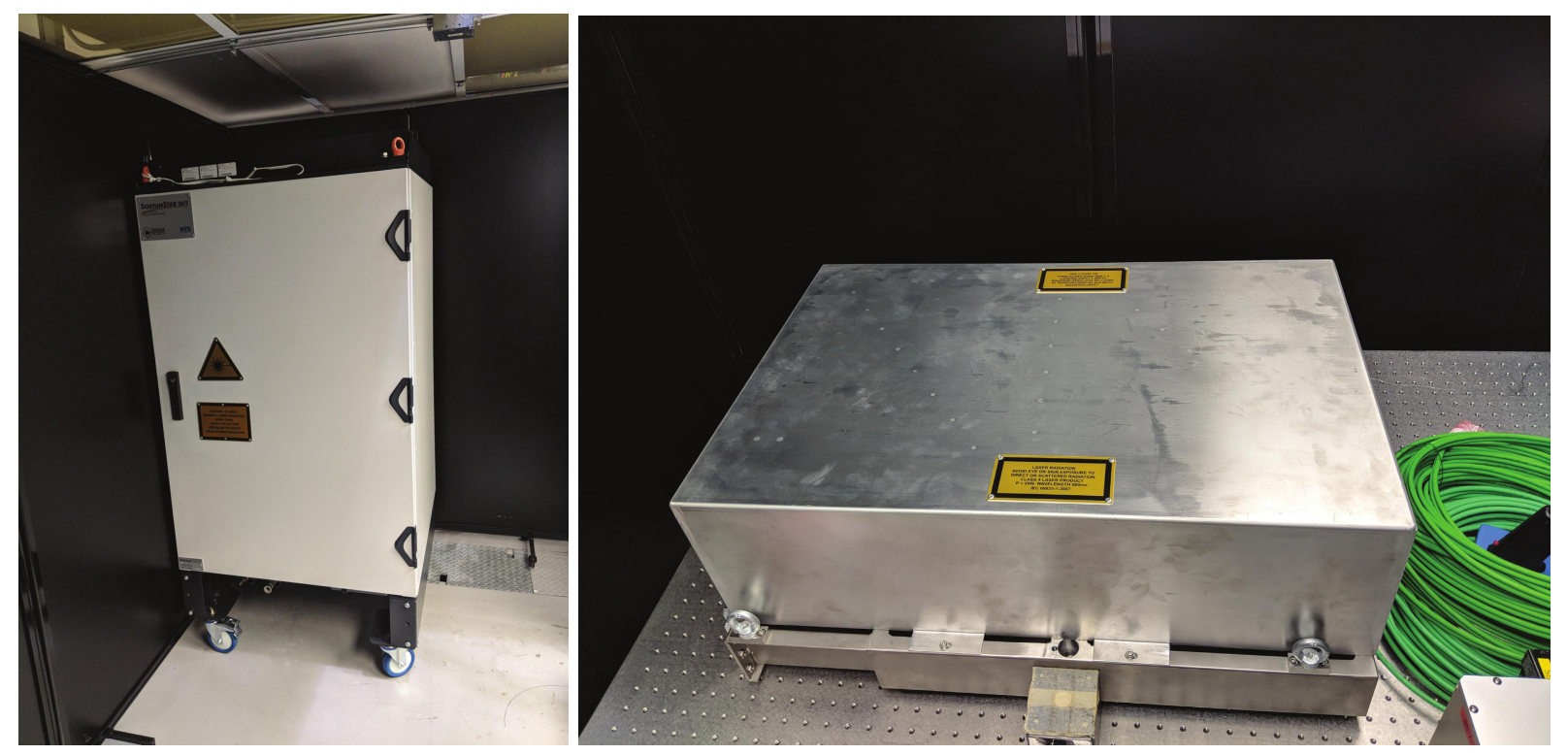

Figure 2 Photos of TOPTICA/MPBC electronics cabinet (left) and laser head (right) in the laser testing lab at the Subaru office. 


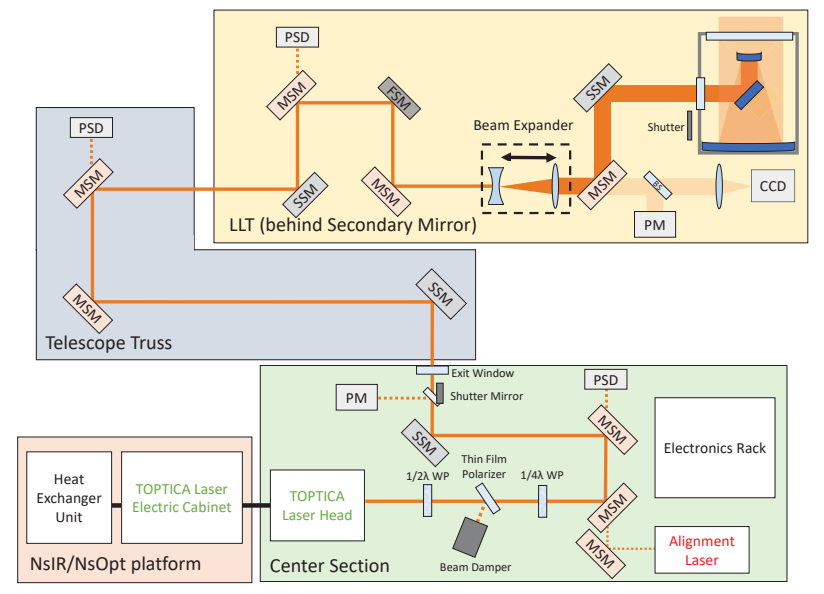

Figure 3 A new Subaru LGS facility configuration. It consists of four sections: 1) TOPTICA/MPBC electronic cabinet and its heat exchange unit on the Nasmyth Infrared platform, 2) TOPTICA/MPBC laser head and an electronic rack on a side of the telescope center section, 3) mirror-based relay along the telescope truss, and 4) tip/tilt mirrors and a beam expander in a LLT side space. MSM, SSM, FSM, PSD, PM are short for manual steering mirror, motorized slow steering mirror, motorized fast steering mirror, position sensitive device, and power meter, respectively.

3) mirror-based relay along the telescope truss, and 4) tip/tilt (TT) mirrors and a beam expander (BE) in a LLT side space. Figure 3 shows what components are included in each sections, and Figure 4 shows where they are located on Subaru Telescope. In the following subsections, we describe components and their requirements of each section.

\subsection{Nasmyth Platform}

TOPTICA/MPBC laser EC and its HEU will be placed on NsIR. The size of the EC is about $1 \times 1 \times 2 \mathrm{~m}$, which is about $1 / 10$ of a space that the current laser unit occupies on NsIR today. When the new EC is installed at the summit, the old laser unit will be removed from NsIR. Currently at NsIR, exchanging IRCS and SCExAO takes a few hours using a crane. The available space will be big enough for two (or even more) instruments to separately located, and we are collaborating with Australian group to design a beam switcher so that we can switch instrument easily during the observation.

In the laser lab at the Hilo office, we are currently using a combination of Thermo Neslab CFT-300 and SMC HRS024-W20 to keep the temperature and the flow rates for both EC and LH. We will test this cooling system when the LH is under the varying gravity to make sure the pressure stays within a tolerance.

\subsection{Center Section}

Three units are located at the center section: 1) a LH, 2) an electronic rack with a laser splicer, and 3) an optical bench (Figure 4). All three are attached on the front side of the center section

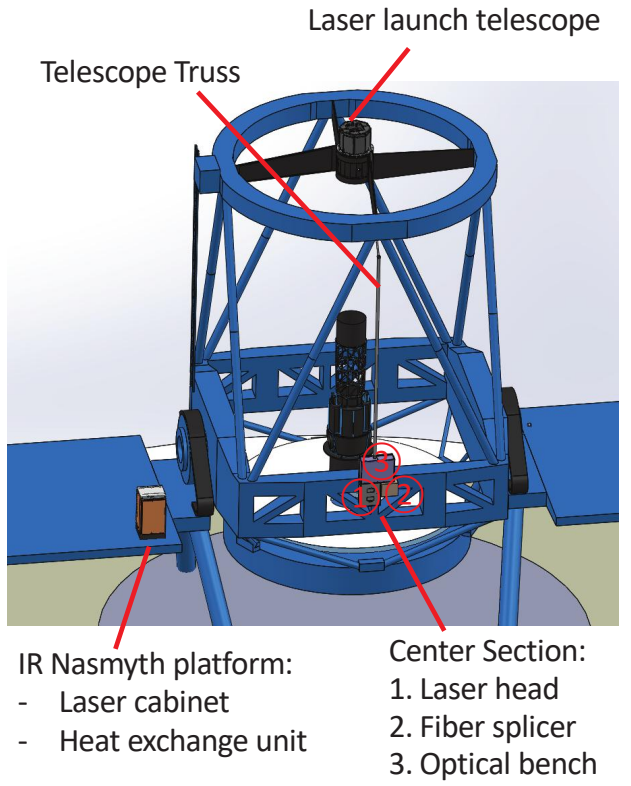

Figure 4 CAD model of Subaru Telescope and preliminary TOPTICA/MPBC laser unit installation plan. 
and will experience varying gravity during observations. A beam from a seed laser installed in the EC on NsIR is sent to the LH by a fiber, and the beam is amplified to 20 to $25 \mathrm{~W}$ inside the LH. After LH, $3 \mathrm{~mm}$ beam is transferred by mirrors. The beam is then sent to the optical bench, and its polarization is controlled by wave plates.

\subsection{Telescope Truss}

The beam has to be transferred to the LLT behind the secondary mirror from the center section using mirrors, and the entire path needs to be covered up for safety purpose. As a result, about $15 \mathrm{~m}$ metallic tube will be laid out along telescope truss.

Two main requirements are 1) a compensation of the telescope deflection and 2) the path of the relay tube. First, there is a maximum deflection of $52 \mu \mathrm{m}$ or 0.7 arcsec between the top ring and the center section when pointing the telescope from the elevation of $90^{\circ}$ to $0^{\circ}$, and motorized slow steering mirrors (SSMs) need to react to the elevation change and compensate the beam displacement. Current design (Figure 3) has three SSMs, one at the exit of the center section, one at the entrance of the telescope truss section, and one at the entrance of the LLT section. Also the deflection of the relay tube itself should not interfere with the beam path, of course.

At Subaru Telescope, the space above the secondary spider cannot be used due to cabling and top unit exchanges, and the relay path need to take under the spider. The relay path should be within the existing shadow of the telescope structure so that it will not affect the current instruments. Figure 5 shows the results seen from the edge of the Nasmyth focus, where

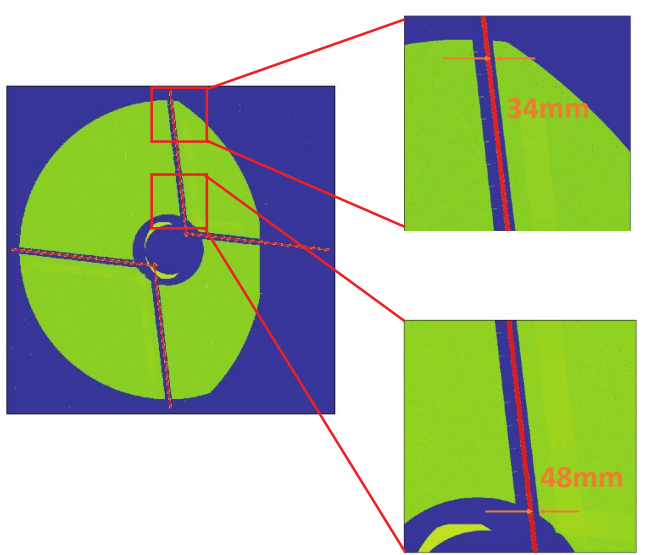

Figure 5 Telescope pupil seen from the edge of the Nasmyth focus field of view. Shadow on the pupil is created by the mirrors attached on the tertiary mirror spider and the center of the secondary mirror (center cone) to avoid the thermal radiation from the telescope structure directly entering in the infrared instruments. The location of the secondary mirror spider is overplotted as red lines. The path of the optical tube that transfer the laser beam to the LLT should be within the shadow. AO instruments are installed. We trace the shadow seeing from all foci using Zemax lay tracing and constrain the size and the path of the relay tube.

\subsection{Laser Launch Telescope}

Our current LLT is a $50 \mathrm{~cm}$ afocal telescope consists of a parabolic primary, a parabolic secondary, and a flat tertiary mirrors (left panel on Figure 6). It expands incoming collimated beam to 12.5 times bigger. The LLT in a square box is sitting in a hexagonal box (right panel on Figure 6), whose side spaces contain optics, electronics, and cables. We will recycle this LLT by completely reorganizing the side spaces. The space indicated by red in Figure 6 will be used for a new set of optics.

A $3 \mathrm{~mm}$ beam sent from the LH all the way to the LLT through the truss is expanded by a BE before entering the LLT. A preliminary design of a BE in the LLT side space and LLT are shown in Figure 6. We are currently developing several replaceable BE units to study how the laser guide star size changes with the launching beam size. The BE units are designed to work with both single (Figure 3) and four (Figure 7) beam configurations (see $\S 3$ for details of four beam configuration). Table 1 shows the analytically calculated beam sizes for different BE magnification, m. $D\left(1 / e^{2}\right)$ is the beam diameter at the LLT secondary mirror (M2) when one beam configuration is used, $D_{2}\left(0.8^{\circ}\right)$ and $D_{2}\left(1.6^{\circ}\right)$ are beam footprint sizes at M2 when the four LGS asterism is $0.8^{\circ}\left(30^{\prime \prime}\right)$ and $1.6^{\circ}$ (60"), respectively, and $D_{b}(s k y)$ is a beam diameter at the exit window (EW) of LLT. With the current LLT, the size of M2 $(D=42 \mathrm{~cm})$ sets the maximum beam size and asterism.

The current design has a motorized fast steering mirror (FSM) before the BE and a motorized SSM after the BE to correct about 1 arcsec on sky TT and about 10 arcsec pointing error between LLT and telescope, 
Table 1. Beam Expander Comparison

\begin{tabular}{ccccc}
\hline \hline $\begin{array}{c}\mathrm{m} \\
\mathrm{m}\left(1 / e^{2}\right) \\
\mathrm{mm}\end{array}$ & $\begin{array}{r}D_{2}\left(0.8^{\circ}\right) \\
\mathrm{mm}\end{array}$ & $\begin{array}{r}D_{2}\left(1.6^{\circ}\right) \\
\mathrm{mm}\end{array}$ & $\begin{array}{c}D_{b}(\text { sky }) \\
\mathrm{mm}\end{array}$ \\
\hline 5.0 & 24.0 & 28.0 & 32.0 & 188 \\
7.5 & 37.5 & 41.5 & 45.5 & 281 \\
8.0 & 40.0 & 44.0 & 48.0 & 300 \\
\hline
\end{tabular}

respectively. Because the FSM need to move fast, it is placed where the beam is still small (thus small optics). The SSM, on the other hand, is placed after so that the required stroke is not magnified by the BE.

\section{SUMMARY AND FUTURE PLANS}

We have received and installed the new laser in the laser lab at the Subaru office in Hilo. The laser power has been confirmed to be $>22 \mathrm{~W}$ in the lab, and some optical components have been ordered. While we are testing off-the-shelf optical components, such as wave plates and glasses for mirrors, in the lab to finalize the choices, we are also finishing our $\mathrm{BE}$ design so that we can order customized lenses for fabrication by the end of this summer. The expected commission of the new laser at the telescope is 2019A semester.

The implementation of the new TOPTICA/MPBC laser is an initial stepping stone toward our ultimate goal, a laser tomography (LT) AO and ground layer (GL) AO, in the next decade. For the LTAO mode, the TOPTICA/MPBC laser beam will be split into four beams, and all four beams go through the same single BE inside the LLT side space. We are expecting to purchase a new LLT for this configuration, and thus the four beams are entering the LLT from the bottom in Figure 7.

The final stage of the new AO development will have four LGSs using two TOPTICA/MPBC lasers and four LLTs (split each beam to two). Figure 8 shows a half of the configuration, and there should be one more of the same thing. LLTs will be located on the center section of the telescope and will create a wide $(D=20 \operatorname{arcmin})$ asterism on sky. With an implementation of a deformable secondary mirror, GLAO mode will be available, whose correction will be 0.2 arcsec over 20 arcmin in K. Please see Ref. 10 and reference therein for details of ULTIMATE Subaru project.
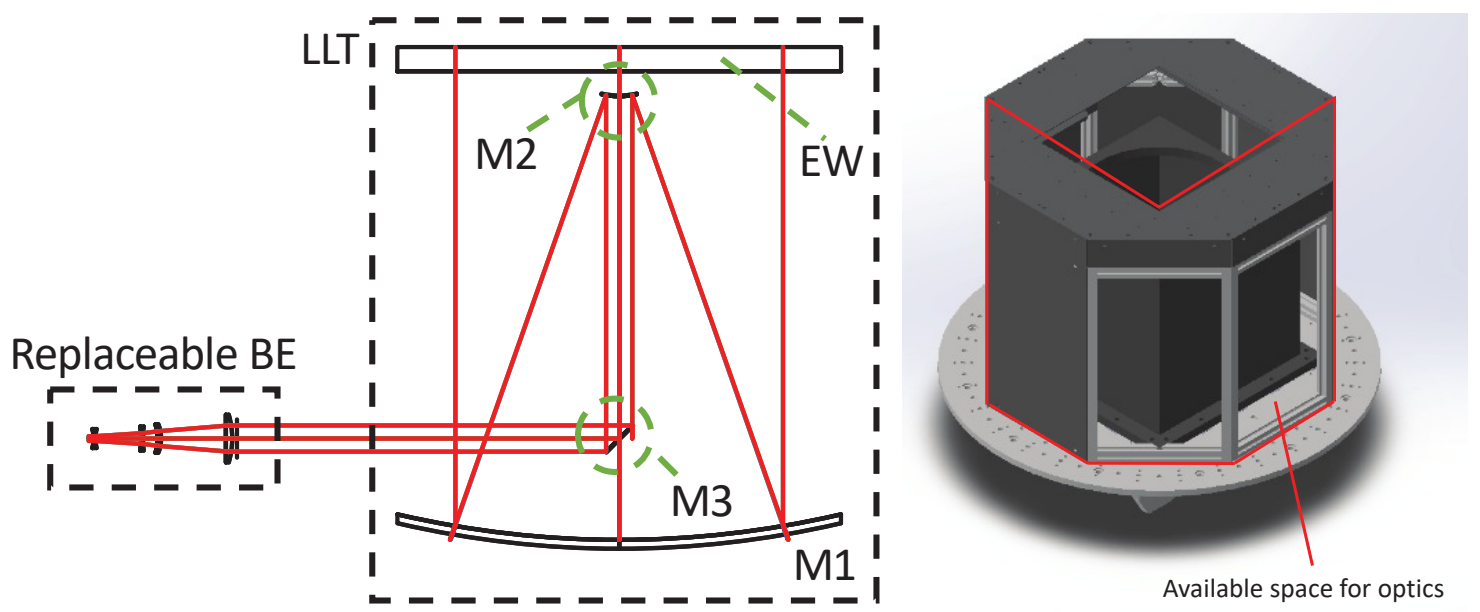

Figure 6 Left: Simplified optical path of a beam expander and the current LLT. Several replaceable BEs (dashed box) will be developed to test which gives the most photons on sky. Right: CAD model of LLT enclosure. The side space will be reorganized and space indicated by red will be used for new optics. 


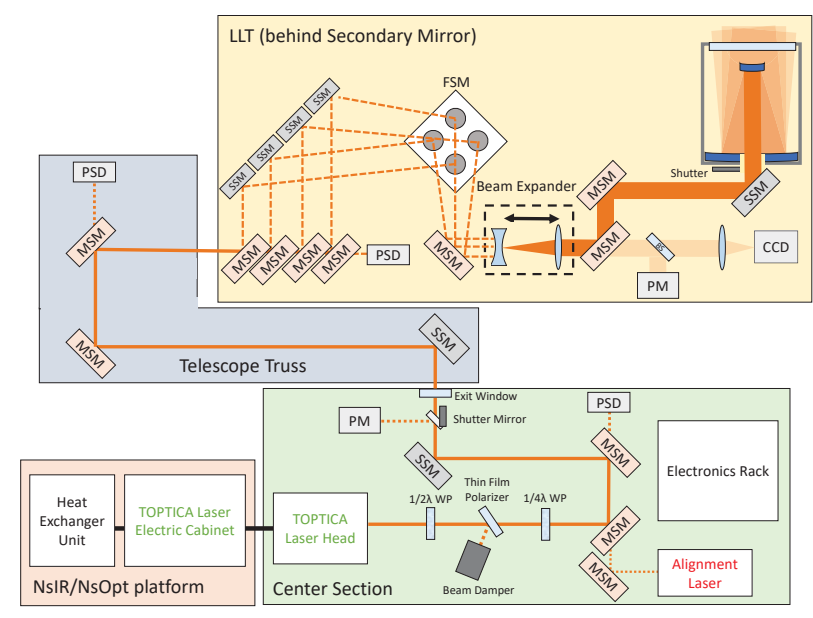

Figure 7 Second stage of the LGS upgrade with the four beam configuration. By launching four beams by one LLT, LTAO mode will be operated.

\section{ACKNOWLEDGMENTS}

The laser guide star upgrade at the Subaru telescope is partly supported by the Japan Society for the Promotion of Science (Grant-in-Aid for Research 17H06129).

\section{REFERENCES}

[1] Rusu, C. E., Oguri, M., Minowa, Y., Iye, M., Inada, N., Oya, S., Kayo, I., Hayano, Y., Hattori, M., Saito, Y., Ito, M., Pyo, T.-S., Terada, H., Takami, H., and Watanabe, M., "Subaru Telescope adaptive optics observations of gravitationally lensed quasars in the Sloan Digital Sky Survey," 458, 2-55 (May 2016).

[2] Kuzuhara, M., Tamura, M., Kudo, T., Janson, M., Kandori, R., Brandt, T. D., Thalmann, C., Spiegel, D., Biller, B., Carson, J., Hori, Y., Suzuki, R., Burrows, A., Henning, T., Turner, E. L., McElwain, M. W., Moro-Martín, A., Suenaga, T., Takahashi, Y. H., Kwon, J., Lucas, P., Abe, L., Brandner, W., Egner, S.,

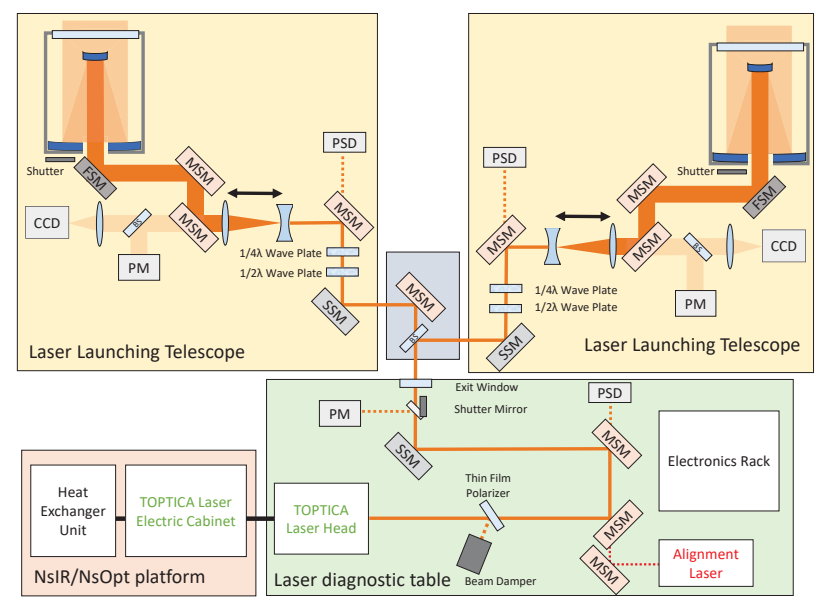

Figure 8 The final stage of the new AO development at Subaru Telescope. With four beams launched from four LLTs in a wide (20 arcmin) asterism, 0.2 arcsec correction over $20 \operatorname{arcmin}$ in $\mathrm{K}$ is expected to be achieved in GLAO mode. 
Feldt, M., Fujiwara, H., Goto, M., Grady, C. A., Guyon, O., Hashimoto, J., Hayano, Y., Hayashi, M., Hayashi, S. S., Hodapp, K. W., Ishii, M., Iye, M., Knapp, G. R., Matsuo, T., Mayama, S., Miyama, S., Morino, J.-I., Nishikawa, J., Nishimura, T., Kotani, T., Kusakabe, N., Pyo, T.-S., Serabyn, E., Suto, H., Takami, M., Takato, N., Terada, H., Tomono, D., Watanabe, M., Wisniewski, J. P., Yamada, T., Takami, H., and Usuda, T., "Direct Imaging of a Cold Jovian Exoplanet in Orbit around the Sun-like Star GJ 504," ApJ 774, 11 (Sept. 2013).

[3] Hayano, Y., Takami, H., Oya, S., Hattori, M., Saito, Y., Watanabe, M., Guyon, O., Minowa, Y., Egner, S. E., Ito, M., Garrel, V., Colley, S., Golota, T., and Iye, M., "Commissioning status of Subaru laser guide star adaptive optics system," in [Adaptive Optics Systems II], 7736, 77360N (July 2010).

[4] Ono, Y. H., Minowa, Y., Clergeon, C. S., Mieda, E., Guyon, O., Lozi, J., Akiyama, M., Rigaut, F., Hayano, Y., and Oya, S., "Ongoing and future AO activities on Subaru telescope," in [Adaptive Optics Systems VI], 10703 (June 2018).

[5] Clergeon, C. S., Minowa, Y., Guyon, O., Ono, Y. H., Mieda, E., Skaf, N., Yoshida, H., Hayano, Y., Tait, P., Hattori, T., and Sahoo, A., "Subaru AO188 upgrade phase 1: integration of the new real-time system ," in [Adaptive Optics Systems VI], 10703 (June 2018).

[6] Saito, Y., Hayano, Y., Saito, N., Akagawa, K., Takazawa, A., Kato, M., Ito, M., Colley, S., Dinkins, M., Eldred, M., Golota, T., Guyon, O., Hattori, M., Oya, S., Watanabe, M., Takami, H., Iye, M., and Wada, S., "589 nm sum-frequency generation laser for the LGS/AO of Subaru Telescope," in [Society of Photo-Optical Instrumentation Engineers (SPIE) Conference Series], 6272, 627246 (June 2006).

[7] Minowa, Y., Hayano, Y., Terada, H., Pyo, T.-S., Oya, S., Hattori, M., Shirahata, M., Takami, H., Guyon, O., Garrel, V., Colley, S., Weber, M., Golota, T., Watanabe, M., Saito, Y., Ito, M., and Iye, M., "Subaru laser guide adaptive optics system: performance and science operation," in [Adaptive Optics Systems III], 8447, 84471F (July 2012).

[8] Chin, J. C. Y., Wizinowich, P., Wetherell, E., Lilley, S., Cetre, S., Ragland, S., Medeiros, D., Tsubota, K., Doppmann, G., Otarola, A., and Wei, K., "Keck II laser guide star AO system and performance with the TOPTICA/MPBC laser," in [Adaptive Optics Systems V], 9909, 99090S (July 2016).

[9] Friedenauer, A., Karpov, V., Wei, D., Hager, M., Ernstberger, B., Clements, W. R. L., and Kaenders, W. G., "RFA-based 589-nm guide star lasers for ESO VLT: a paradigm shift in performance, operational simplicity, reliability, and maintenance," in [Adaptive Optics Systems III], 8447, 84470F (July 2012).

[10] Rigaut, F., Minowa, Y., Akiyama, M., Ono, Y. H., Watanabe, T., Korkiakoski, V. A., Clergeon, C. S., d'Orgeville, C., Gausachs, G., Herrald, N., Mieda, E., Tanaka, Y., Iwata, I., Tanaka, I., Takato, N., Arimoto, N., Yoshida, M., Kodama, T., Hayano, Y., Oya, S., and Motohara, K., "A conceptual design study for Subaru Ultimate GLAO," in [Adaptive Optics Systems VI], 10703 (June 2018). 